\title{
Preliminary Assessment of Low-cost Unmanned Aerial Systems (UAS) for Creating Aerial Photographs for Natural Resource Research
}

\author{
Eric Compas $^{1}$ \\ Department of Geography, Geology, and Environmental Science \\ University of Wisconsin-Whitewater \\ Whitewater, WI
}

\begin{abstract}
The recent significant reduction in the cost of unmanned aerial systems (UAS) has expanded access to this technology to researchers in a variety of fields. For aerial mapping, UAS holds the promise to substantially increase the resolution of imagery and provide better control over when imagery is produced. In the natural resources, UAS could greatly expand the type of research questions that can be asked at a relatively low cost. This research assessed the utility and characteristics of low-cost UAS aerial photography for natural resource applications. Four craft, commercial fixed-wing and multirotor and handbuilt fixed-wing and multirotor, were tested in this study. Our findings conclude that UAS aerial photography is approximately $10 \mathrm{x}$ more detailed than traditional aerial photography, but issues with spatial accuracy and available flight weather may limit its use for some applications.
\end{abstract}

\section{Introduction}

Significant technological improvements in microcontrollers and batteries and lower overall costs have increased the access to unmanned aerial systems (UAS), also known as "drones," to civilian and research communities (Samant and Datta 2014). Increased energy densities of batteries, particularly lithium-ion based formulas, and the reduced size and broader access to inertial measurement units (IMU) and high-accuracy global positioning system (GPS) units has allowed both commercial and open source communities to produce UAS at much lower cost with increased capabilities. For example, the on-paper capabilities of a $\$ 25,000-40,000$ system just a few years ago are currently available for around $\$ 3,000$. This reduction in costs substantially increases the general availability of UAS to researchers in a broad set of fields. Furthermore, given the ready availability of UAS components, researchers have the option of constructing their own UAS for as little as $\$ 500$.

Potential advantages of UAS-derived aerial photography including producing imagery at a time desired by the research, e.g. release of a tracked animal, to repeat imagery fairly frequently, e.g. track the senescence of vegetation in the fall, and produce high resolution and detailed imagery.

\footnotetext{
${ }^{1}$ The author would like to acknowledge the support of the Wisconsin Space Grant Consortium (WSGC) Research Infrastructure program in conducting this research.
} 
With commercial UAS and consumer-grade cameras, imagery with $1 \mathrm{~cm}$ resolution is possible over small areas. In addition, the UAS can be deployed on short notice to capture events such as natural disasters or other unexpected phenomenon. These advantages are significant compared to conventional aerial photography which requires substantial planning and cost and is often captured, at most, once a year in most places.

Given the rapid evolution of available UAS technology, general assessments and limitations of UAS imagery from just a few years ago (e.g. Zongjian 2008) are no longer relevant. Accordingly, the purpose of this research is to ascertain the characteristics and limitation of UAS-derived aerial photography using contemporary, generally available systems. This assessment includes determining the limits of spatial accuracy, image resolution, and aerial coverage along with anecdotal observations on field use of the equipment. We investigated these characteristics for four craft, commercial fixed-wing and multirotor UASes and handbuilt fixed-wing and multirotor UASes, and post-processed our imagery with both commercial and opensource software. For this study, we did not investigate the spectral characteristics of the consumer-grade cameras typically used on low-cost UAS since we were largely interested in true-color, traditional aerial photography for visual analysis (see Hunt et al. 2010 and Rabatel et al. 2012).

\section{Background}

UAS technology has seen widespread testing for natural applications, with research in areas such as wildlife research (Jones IV et al. 2006), rangeland monitoring (Laliberte et al. 201), and natural hazard mapping (Hirokawa et al. 2007). In general, these studies have shown that UAS imagery is relatively inexpensive, flexible in its acquisition time, and often of better quality than traditional image sources. Additionally, the technology potentially allows for the posing of novel research questions in many of these areas.

A UAS for aerial mapping comprises multiple, synchronized components to successfully complete a mission. Field components include the airframe with motors and control surfaces, flight controller, a GPS, a redundant radio system, and a camera, along with a ground control station with a computer and RC-style transmitter (for manual flight). The ground control software allows for the generation of pre-planned flight path the craft will follow with significant allowance for both end-to-end and side-to-side overlap of photos. Once launched, the craft is switched into an autonomous mode and flies the preplanned route. Imagery is taken throughout the flight path either at a predetermined interval, e.g. once every three seconds, or a set distance. Fixed-wing aircraft require manual landing while multirotor craft can both launch and land autonomously. The result of the mission is between tens to hundreds of images to be processed.

Post-processing and merging imagery is required in order to generate a usable mosaic of the area of interest. A technique from computer vision, called structure-from-motion (SfM), is used as an 
effective tool to simultaneously merge large sets of imagery and create an elevation model of the surface (Westoby et al. 2012). The result of the SfM processes is a single orthorectified image for use in geographic information systems (GIS) and/or remote sensing software packages for further analysis.

Within the USA, Federal Aviation Administration (FAA) rules regarding drones, while permissive, do present some limitations for the use of UAS for research (Rango and Laliberte 2010). Craft are required to fly below 400' above ground level, within visual line of sight, and cannot be used over populated areas and within certain controlled airspaces, e.g. large airports. In addition, some states have imposed additional regulations, largely to protect privacy. For most natural resource applications, these current regulations do not pose significant limitations. The FAA currently has draft regulations for UAS that are likely to change this legal framework substantially in 2016 .

\section{Methods}

Our methods included the acquisition of both fixed-wing and multirotor commercial UAS, purchasing parts and building two UAS from scratch, conducting field trials of all four units, post-processing imagery from successful flights, and characterizing the image characteristics from each.

We purchased two commercial units: an E384 fixed-wing system from Event38 (www.event38.com) for \$2,500 which included two consumer-grade Canon S100 cameras and a SOLO quadcopter from 3D Robotics ( HERO4 Silver for a camera and a stabilizing gimbal. Both units use versions of an open source autopilot project, ArduPilot or APM, as flight controllers. Additionally, the components for APM-based fixed-wind and quadcopter platforms were purchased from a variety of vendors costing $\$ 450$ and $\$ 550$, respectively, not including camera costs. A separate, mirrorless, higher resolution camera, a Sony a5100 with a fixed $16 \mathrm{~mm}$ lens, was purchased (\$700) to test on all platforms. Being APM-based, both commercial and hand-built units were compatible with a ground station control software called Mission Planner used in all testing. Autonomous missions, including aerial photography survey grids, were planned and carried out with these UAS combinations. We received formal training on our first purchase, the E384 fixed-wing craft, from Event38 which included the somewhat difficult task of manually landing the plane.

For processing our imagery, we used two different workflows, both utilizing structure-from-motion (SfM) photo stitching software. One path utilized a combination of open source tools including VisualSFM (Wu 2014) and CMPMVS (Jancosek 2012) to create an orthorectified and stitched aerial photograph. The second used a commercial package, Pix4D ( $\$ 1,990$ for an educational license), to carry out the same workflow. 
Three test sites were identified that had sufficient landing space for the fixed-wing craft (an approximately $100 \mathrm{~m}$ by $30 \mathrm{~m}$ obstruction-free strip), grassland and forest vegetation, and human structures. At these sites, a series of flights were flown varying the following: with and without differential GPS ground control, with each of the available camera platforms, and at various altitudes $(20,40,60$, and $80 \mathrm{~m}$ above ground level). After each flight, the resulting imagery (ranging from 150 to 700 separate photographs) was processed with both open source (VisualSFM/CMPMVS) and commercial (Pix4D) packages and the results were compared. At all altitudes we used a ground target to ascertain the practical image resolution of the pre- and post-processed aerial photographs (see Figure 1).

We quantified image quality both qualitatively through visual inspection and quantitatively through the final image cell resolution and through observing a standardize ground target to determine the ground resolved distance (Cambell 2002, 275), a standard measure of image spatial resolution. The ground target contains squares from 1 to $7 \mathrm{~cm}$ in size rotated at $0^{\circ}, 22.5^{\circ}$, $45^{\circ}$, and $67.5^{\circ}$ (see Figure 1). For quality testing, we used the 3DR Solo and the Sony a5100 camera allowing us to minimize horizontal speed of the camera and maximizing resolution.
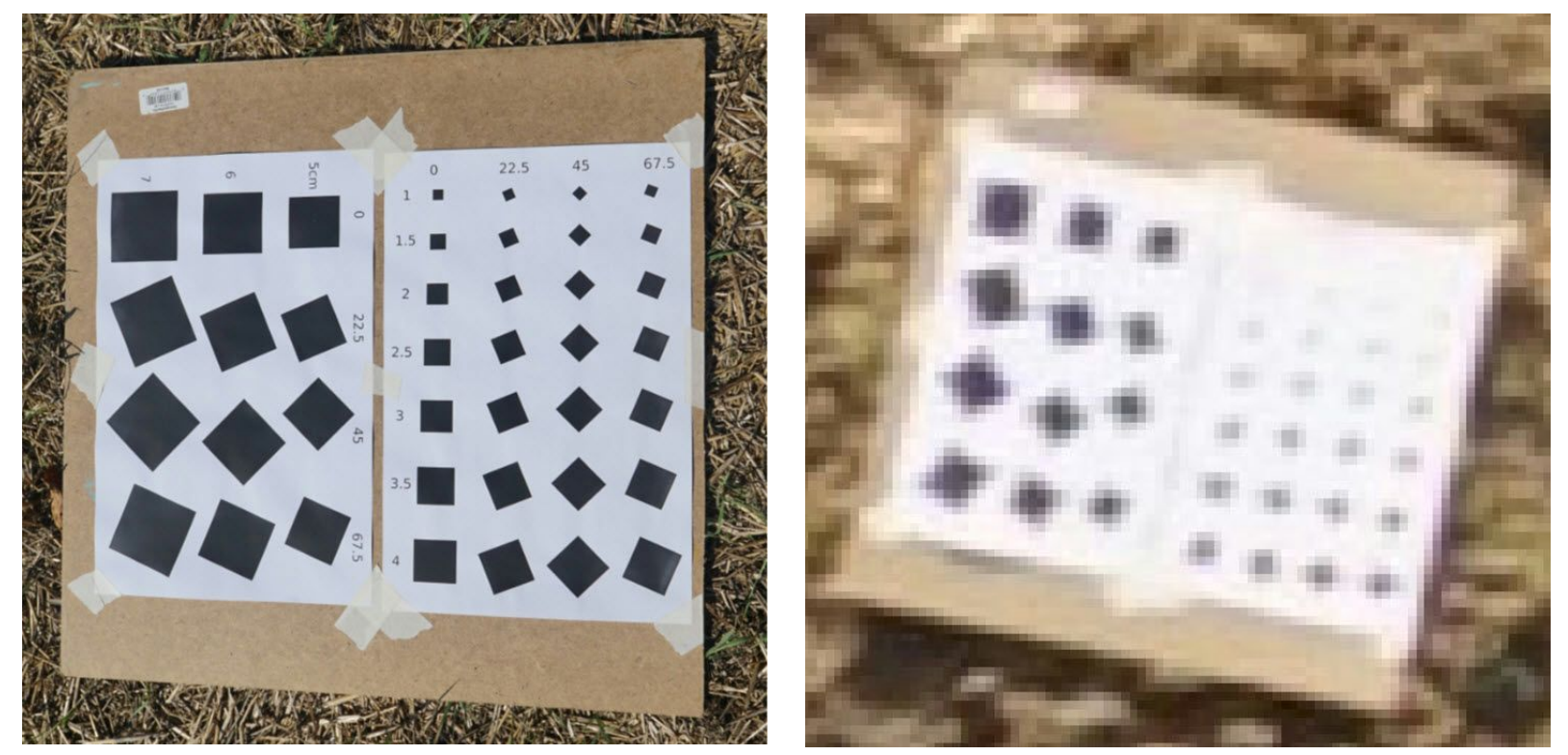

Figure 1. Ground resolved distance target seen from ground (left) and $40 \mathrm{~m}$ altitude (right).

The theoretical image cell resolution was calculated using simple trigonometry from the camera sensor size and resolution while the average cell resolution was reported by the Pix 4D software (cell resolution varies due to changing elevations and camera angles across the study area). The ground resolved distance was measured by visual inspection of the ground target in unprocessed JPEG images and the final orthorectified image mosaic at each flight altitude. A size was determined to be "resolved" if all squares in a given row were visible. So, for the $40 \mathrm{~m}$ altitude image in Figure 1, the ground resolution distance was $2.0 \mathrm{~cm}$. 
The spatial accuracy, the correct placement of the image on earth's surface, was calculated through the use of differential GPS. Prior to flying, a set of markers (bright orange soccer cones) were spread within and around the area as ground control points to be imaged and locations determined using a Trimble GeoXH GPS unit. Using ESRI's ArcGIS software, these locations were compared with the visible markers in the Pix4D orthorectified image (which uses the GPS locations at which images are taken) and the distance between these locations measured. In addition, both the Pix4D and VisualSFM images were georeferenced using these ground control points in ArcGIS using an affine, or first order polynomial, transformation. The georeferencing tool provides a measure of how well the resulting image fits the provided ground control points.

Given that our goal was to create aerial photographs for visual interpretation, we did not measure or explore the spectral resolution of the resulting imagery or assess our imagery for traditional remote sensing techniques such as image classification or measurements of vegetation health. Others (e.g. Hunt et al. 201) have noted the poor spectral resolution and overlap between neighboring bands in consumer grade cameras that limit utility for direct physical measurements.

Finally, we collected anecdotal information on the field experiences of our flights and compiled a list of advantages and disadvantages of the platforms we used and the overall process of UAS image acquisition.

\section{Results}

We successfully flew and carried out autonomous missions with all four UAS platforms. However, only with the two commercial platforms were we able to consistently carry out aerial mapping missions. With the commercial platforms, we were able to carry out all of our test flights to assess image quality and accuracy with details provided below.

With all our platforms we experienced uncontrolled and anomalous flights which resulted in "crashes" with all platforms. While the commercial units needed minor repairs, e.g. replacing a broken propellor, the handbuilt units required major repairs including replacement components, e.g. complete quadcopter frame. In addition, the uncontrolled flights of the handbuilt platforms raised safety concerns. In one instance, the quadcopter experienced a "fly away" and flew approximately 2,000 m off course, passed a preset "geofence," across a state highway, and crashed after the pilot switched to manual control and turned off the throttle. Given these safety concerns and the potential to damage expensive cameras, we did not complete mapping missions with the handbuilt units within our research timeframe. 

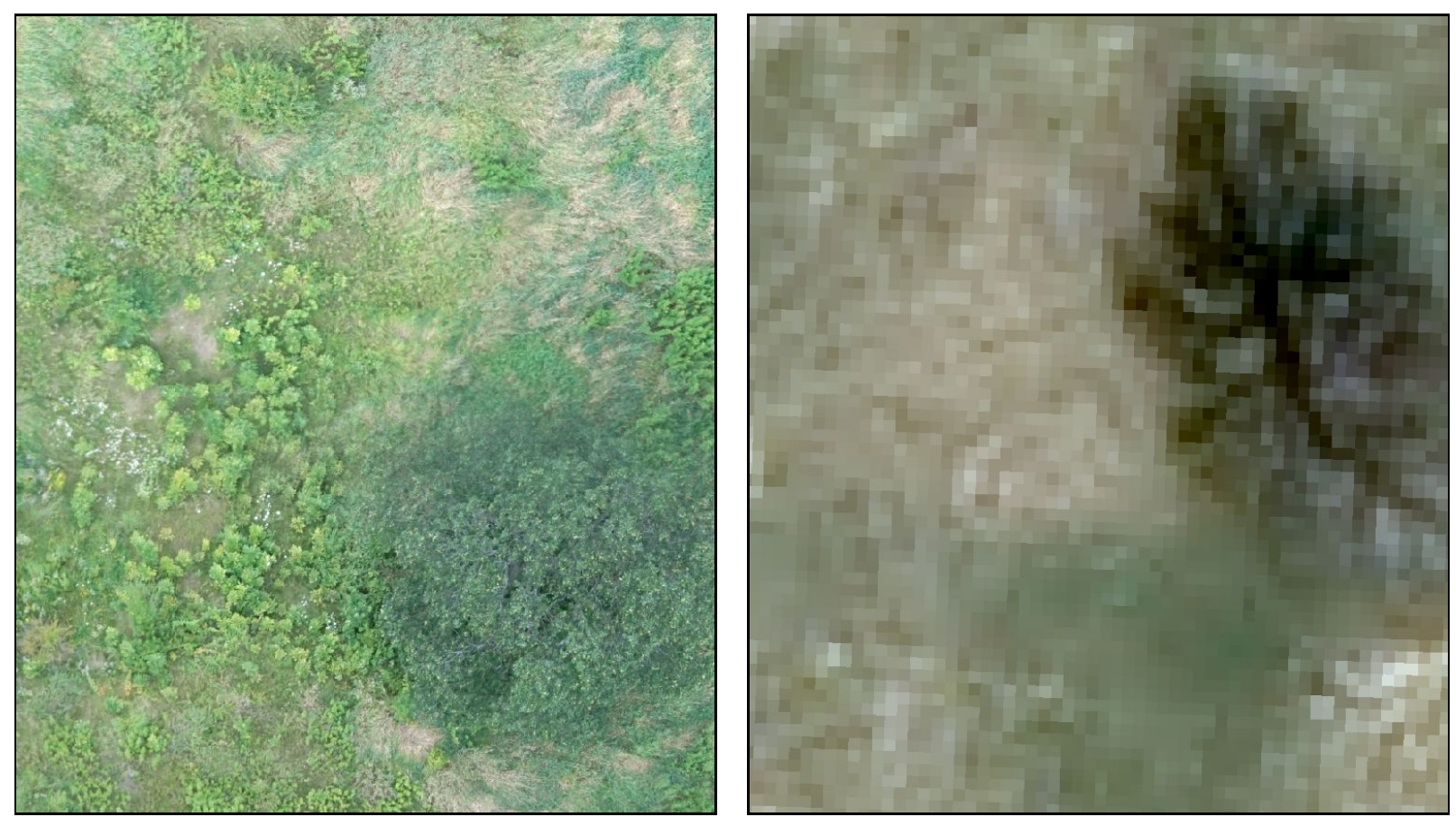

Figure 1. Example imagery of same location with $1.6 \mathrm{~cm}$ resolution UAS imagery on left and $46 \mathrm{~cm}$ resolution 2012 Wisconsin Regional Orthophotography Consortium (WROC) imagery on right (source WisconsinView).

We were successful in completing missions both with the fixed-wing and multirotor craft. Being fully autonomous, the multirotor proved much simpler to deploy and could be launched from any site requiring only a $1 \times 1$ meter space to launch and land. The disadvantage was the mapping area that could be covered. At $80 \mathrm{~m}$ altitude, approximately 16 hectares ( 40 acres) could be mapped per battery. Since the fixed-wing required manual landing, a large landing strip (approx. $30 \mathrm{x}$ $100 \mathrm{~m}$ ) aligned with the current wind direction and with the opportunities for "go arounds" was necessary. At two out of our three sites, the landowner mowed special landing strips for us to us. The advantage of the fixed-wing was a substantial increase the mapping area with up to 120 hectares (300 acres) per flight.

For a subset of our imagery, we post-processed using both the open source VisualSFM and the commercial Pix4D. While both were successful in generating usable image mosaics, there was substantial differences in the processing procedure and the resulting image quality of each. The open source tools were much more likely to crash, could not handle more than 500 images within a set, and even with substantial match points between overlapping images, often left unprocessed holes in the middle of our study areas. Additionally, the open source tools resampled the source imagery resulting in a resolution one-quarter that of Pix $4 \mathrm{D}$, e.g. from $1 \mathrm{~cm}$ to $4 \mathrm{~cm}$. Accordingly, we utilized Pix4D for most of our postprocessing work.

Table 1 shows the result of our image resolution measurements using the commercial Pix4D 
package. In general, our average cell resolution as reported by Pix4D was close to the theoretical cell resolution across all altitudes. As for ground resolution distances, a general rule of thumb is that objects of twice the cell size are generally visible - a theoretical ground resolution distance. We found that for lower altitudes, this relationship held for unprocessed images. However, this distance increased within the processed (orthorectified and mosaiced) imagery and with higher altitudes. While the process of merging multiple overlapping images is likely to reduce overall resolution, it is unclear why the increase in altitude led to a loss in resolution.

Table 1. Theoretical and observed image resolution and ground resolution distance.

\begin{tabular}{|c|c|c|c|c|c|}
\hline $\begin{array}{l}\text { Flight } \\
\text { altitude }\end{array}$ & $\begin{array}{l}\text { Theoretical } \\
\text { cell resolution } \\
(\mathrm{cm})\end{array}$ & $\begin{array}{l}\text { Pix4D average } \\
\text { cell resolution } \\
(\mathrm{cm})\end{array}$ & $\begin{array}{l}\text { Theoretical } \\
\text { ground resolution } \\
\text { distance }(\mathrm{cm})\end{array}$ & $\begin{array}{l}\text { Observed } \\
\text { unprocessed ground } \\
\text { resolution distance } \\
\text { (cm) }\end{array}$ & $\begin{array}{l}\text { Observed Pix4D } \\
\text { orthorectified ground } \\
\text { resolution distance } \\
\text { (cm) }\end{array}$ \\
\hline $20 \mathrm{~m}$ & 0.49 & 0.54 & 0.98 & 1.5 & 1.5 \\
\hline $40 \mathrm{~m}$ & 0.98 & 0.99 & 1.96 & 2.0 & 3.0 \\
\hline $60 \mathrm{~m}$ & 1.47 & 1.53 & 2.94 & 3.0 & 6.0 \\
\hline $80 \mathrm{~m}$ & 1.96 & 2.03 & 3.92 & 5.0 & 6.0 \\
\hline
\end{tabular}

Anecdotally, the resolution provided by the imagery from all altitudes provides substantially more information and detail than is visible (approximately by one order of magnitude, 10x) in traditionally available 1 or $2 \mathrm{~m}$ resolution imagery available through programs such as the National Agriculture Imagery Program (NAIP). Individual plants and trees are visible, allowing for detailed distribution and extent metrics to be calculated and compared through time. The 20 $\mathrm{m}$ and $40 \mathrm{~m}$ imagery provide nearly two orders of magnitude (100x) the resolution of traditional aerial photography.

For spatial accuracy, an image with a significant number of ground control points $(n=13)$ was used to demonstrate the spatial accuracy of UAS imagery. The image was taken with the fixed wing aircraft using the Canon s100 camera with built-in GPS at an $80 \mathrm{~m}$ altitude. The measured root-mean-squared (RMS) error between the ground control points in the image and differential GPS was $2.25 \mathrm{~m}(n=13)$. After an affine transformation was applied using all 13 points, this RMS error was reduced to $0.46 \mathrm{~m}$. Note that the direction of the ground control errors were not consistent. This means the spatial inaccuracy is not systematic and, therefore, errors are the result of shifts in different directions throughout the image. Any attempt to increase accuracy beyond the $0.46 \mathrm{~m}$ would require a higher-order transformation, i.e. "rubber sheeting," with substantially more ground control points. These inaccuracies, particularly in the image without the affined transformation applied, raise concerns about the use of UAS repeat photography to compare patterns and distributions through time. The $2.25 \mathrm{~m}$ error is 110 times larger than the average cell resolution for this altitude. Any fine-scale mapping and change detection of vegetation extents or patterns would have to account for such spatial inaccuracies. 
Other anecdotal observations from our UAS mapping include suggested field procedures and safety, the vagaries of weather, the limited mapping area, and the challenges of building a UAS from scratch. First of all, safety did not emerge as a significant issue with our UAS field experiences. In general, we followed the Academy of Model Aeronautics' Aircraft Safety Code (AMA 2014) and guidelines for autopilot system (AMA 2013). We also established standard procedures for pre-flight preparations, e.g. charging batteries, and pre-flight checklists, e.g. control surface tests. One site was within 5 miles of a local airfield, and we contacted both commercial operators at the airport well before conducting flights. Additionally, we did have two occasions when a small aircraft entered the area in which we were mapping. On both occasions, while there was a substantial difference in the altitude of our craft and the occupied aircraft, we still quickly shifted to manual override and lowered the plane to nearly ground level. The most significant safety concern we encountered was the safe charging and storage of the lithium phosphate batteries used in small UAS. They can self ignite if damaged in a crash, are overcharged, or are undercharged. Most UAS batteries do not contain the over- and under-charge protection circuitry common on other consumer grade electronics. While encountering no fires, we damaged and retired three batteries during the year-long course of this grant.

Second, while theoretically one could sample a study area at a fixed interval for change detection, e.g. once per week, the weather does not always cooperate. Ideally, all images in a sequence would be taken at a fixed interval, at the same time of day (or same sun angle), and with little to no cloud cover or haze. In addition, the wind during this time frame needs to be 15 mph or less. In reviewing this last summer's weather, we found that only 7 days from May 1 to August 31 that met this criteria. ${ }^{2}$

Third, we found that the area we wanted to map was constrained both by the area that can be mapped on a given set of flight batteries and within the FAA requirement of keeping the aircraft in sight at all times. With the fixed-wing craft, we were able to consistently map around 120 hectares (300 acres) per flight, while with the quadcopter, we could only map 16 hectares (40 acres) per flight. Of our three study sites, none could be mapped in a single flight. All required multiple flights, which dramatically increased time in field and often resulted in setup errors or changing weather conditions. Additionally, some portion of each study area required moving to a new location to enable observation of the craft in that portion of the site and, in one study location, one portion of the site was unreachable due to insufficient observation points.

Finally, we found that building a UAS from scratch meant spending a considerable amount of time on the technology itself, rather than using it for mapping. To build an effective UAS, one needs to understand flight dynamics, propellor and motor options, connection various microcontroller devices and the electronic limitations and requirements of each, and the ability to

\footnotetext{
${ }^{2}$ Based on Madison, WI raw METAR data within this time period.
} 
troubleshoot when the inevitable issue emerges. The cost-effectiveness of scratch building a UAS is mitigated once one has purchased additional parts for fixing mishaps and in calculating the value in one's time in learning the technology and building a unit. Unless a research project required customized UAS functionality, e.g. RFID readers or collision avoidance systems, the off-the-shelf commercial units were much more cost-effective for collecting usable imagery.

\section{Conclusions}

Given the goal of producing high resolution and spatially accurate aerial photography from low-cost UAS "drones," this research project was successful in creating photography and measuring its general characteristics. This background research in UAS technology will better allow other researchers to design studies based on UAS technology and assess the utility of UAS technology to address their specific research questions. In general, aerial mapping with UAS allows for at least an order of magnitude (and nearly two) increase in the resolution of aerial photography available for natural resource applications. Given the additional researcher control over when and where imagery is taken, UAS mapping represents a new, potent, low-cost tool for assessing natural resource patterns and changes. Given our experience with both commercial and built-from-scratch UAS, we recommend commercial units for most mapping applications.

The technology, though, has important caveats for adoption by a potential researcher. The technology requires a substantial time commitment to become properly trained and gain an understanding of effective mapping and safety procedures. Additionally, depending on the study region, weather may prove a confounding factor and limit repeat photography for change detection. Finally, the spatial accuracy of imagery is relatively low if not combined with expensive differential GPS equipment.

\section{Acknowledgements}

In addition to WSGC, I would like to thank all of the Advanced GIS students, Aaron King and Tyler Tomaszewski in particular, who helped with the building and testing of our UAS program.

\section{References}

Academy of Model Aeronautics (AMA). 2013. Radio Controlled Model Aircraft Operation Utilizing Failsafe, Stabilization and Autopilot Systems (AMA Document \#560). Available at http://www.modelaircraft.org/files/560.pdf (accessed May 15, 2014).

Academy of Model Aeronautics (AMA). 2014. AMA National Model Aircraft Safety Code. Available at https://www.modelaircraft.org/files/105.pdf (accessed May 15, 2014).

Campbell, J. 2002. Introduction to Remote Sensing. 3rd ed. New York, NY: CRC Press. pp. 654.

Hirokawa, R., Kubo, D., Suzuki, S., Meguro, J., and Suzuki, T. 2007. "A Small UAV for 
Immediate Hazard Map Generation.” In AIAA Infotech@Aerospace 2007 Conference and Exhibit. American Institute of Aeronautics and Astronautics. Accessed August 7, 2015. http://arc.aiaa.org/doi/abs/10.2514/6.2007-2725.

Hunt, E.R., Hively, W.D., Fujikawa, S.J., Linden, D.S., Daughtry, C. and McCarty, G.W. 2010. "Acquisition of NIR-Green-Blue Digital Photographs from Unmanned Aircraft for Crop Monitoring." Remote Sensing 2 (1): 290-305.

Jancosek, M. and Pajdla, T. 2012. Multi-View Reconstruction Preserving Weakly-Supported Surfaces. IEEE Conference on Computer Vision and Pattern Recognition 2011. http://cmp.felk.cvut.cz/ jancom1/JancosekCVPR11.pdf. Accessed June 5, 2014.

Jones IV, G.P., Pearlstine, L.G. and Percival, H.F. 2006. "An Assessment of Small Unmanned Aerial Vehicles for Wildlife Research.” Wildlife Society Bulletin 34 (3): 750-58.

Laliberte, A.S., Herrick, J.E., Rango, A. and Winters, C. 2010. "Acquisition, Orthorectification, and Object-Based Classification of Unmanned Aerial Vehicle (UAV) Imagery for Rangeland Monitoring.” Photogrammetric Engineering \& Remote Sensing 76 (6): 661-72.

Rabatel, G., Gorretta, N. and Labbe, S. 2012. "Getting Simultaneous Red and near Infrared Bands from a Single Digital Camera for Plant Monitoring Applications." In . Valencia, Spain. http://cigr.ageng2012.org/images/fotosg/tabla_137_C1122.pdf.

Rango, Albert, and Andrea S. Laliberte. 2010. "Impact of Flight Regulations on Effective Use of Unmanned Aircraft Systems for Natural Resources Applications." Journal of Applied Remote Sensing 4 (1): 043539-043539.

Samant, H., and Datta, A. 2014. "Domesticating the Drones." Geospatial World. 5 (5):34-40.

Westoby, M. J., J. Brasington, N. F. Glasser, M. J. Hambrey, and J. M. Reynolds. 2012. “'Structure-from-Motion' Photogrammetry: A Low-Cost, Effective Tool for Geoscience Applications." Geomorphology 179: 300-314.

Wu, C. 2014. VisualSFM: A Visual Structure from Motion System. http://ccwu.me/vsfm Accessed June 5, 2014.

Zongjian, L. 2008. "UAV for Mapping — low Altitude Photogrammetric Survey." International Archives of Photogrammetry and Remote Sensing, Beijing, China. http://www.isprs.org/proceedings/XXXVII/congress/1_pdf/202.pdf. 